\title{
Planck-scale effects on Bose-Einstein condensates
}

\author{
F. Briscese ${ }^{1,2}$ (a), M. Grether ${ }^{3}$ (b) and M. DE Llano ${ }^{4}$ (c) \\ 1 Escuela de Física, Universidad Industrial de Santander - Ciudad Universitaria, Bucaramanga 680002, COLOMBIA \\ 2 Istituto Nazionale di Alta Matematica Francesco Severi, Gruppo Nazionale di Fisica Matematica - Piazzale Aldo \\ Moro 5, c.a.p. 00185, Rome, ITALY \\ 3 Facultad de Ciencias, Universidad Nacional Autónoma de México - 04510 México, DF,MEXICO. \\ 4 Instituto de Investigaciones en Materiales, Universidad Nacional Autónoma de México - 04510 México, DF, \\ MEXICO. \\ PACS 04.60.-m - Quantum gravity \\ PACS 04.60.Bc - Phenomenology of quantum gravity \\ PACS $04.90 .+e-$ Other topics in general relativity and gravitation
}

\begin{abstract}
The effects of a Planck-scale deformation of the Minkowski energy-momentum dispersion relation on the phenomenology of non-trapped Bose-Einstein condensates (BECs) are examined. Such a deformation is shown to cause a shift in the condensation temperature $T_{c}$ of the BEC and, for a specific functional form of deformation, this shift can be as large as the current measured precision on $T_{c}$. For a ${ }_{37}^{85} R b$ cold-atom BEC with a particle density $n \simeq 10^{12} \mathrm{~cm}^{-3}$ we find a fractional shift of order $10^{-4}$, but this can be much larger for even more dilute BECs. We discuss the possibility of planning specific experiments with BECs that might provide phenomenological constraints on Planck-scale physics. These corrections to $T_{c}$ are found to be extremely small for ultrarelativistic BECs implying that, in some cases, Planck-scale effects may be more important in low- rather than high-energy processes.
\end{abstract}

Lively interest has recently emerged to experimentally probe quantum-gravitational Planck-scale effects [1] due to deformations of the standard Minkowski free-particle energy-momentum dispersion relation of special relativity. Such deformations are a general feature of quantumgravity theories, as e.g., loop quantum gravity [2 or noncommutative geometries 9-12. Further, it is also of great relevance in the general context of Lorentzsymmetry breaking [13. Quite generally, a deformed dispersion relation can be written as

$$
E(p) \equiv E_{0}(p)+\delta E\left(p, m, M_{P}\right)
$$

where $E_{0}(p) \equiv \sqrt{p^{2} c^{2}+m^{2} c^{4}}$ is the familiar Minkowski dispersion relation, with $p$ the particle momentum, $m$ its rest mass and $c$ the speed of light. Here $\delta E\left(p, m, M_{P}\right)$ represents the deviation from $E_{0}(p)$ which in addition is

\footnotetext{
(a) E-mail: briscese.phys@gmail.com

(b) E-mail: mdgg@hp.fciencias.unam.mx

(c) E-mail: dellano@unam.mx
}

a function of the Planck mass $M_{P}$ while its explicit form depends on the details of the quantum-gravity model used. The relation (1) is assumed to be universal, i.e., is the same for all the elementary particles, including composite particles such as nucleons or atoms when internal degrees of freedom are negligible.

The dependence on the Planck mass $M_{P}$ is introduced since one expects that departures from Lorentz symmetry become important at Planck scales, but one should require that this symmetry be restored when $p \ll c M_{P}$. Moreover one would like to preserve the interpretation of $m$ as the particle rest mass. In general, therefore, one should impose the two conditions

$$
\begin{aligned}
& \delta E\left(p=0, m, M_{P}\right)=0 \\
& \delta E\left(p, m, M_{P}\right) \underset{M_{P} \longrightarrow \infty}{\longrightarrow} 0 .
\end{aligned}
$$

Initially, attempts to constrain the functional form of $\delta E\left(p, m, M_{P}\right)$ were in an astrophysical context where par- 
ticles are in the ultrarelativistic (UR) regime, $p \gg m c$ 14. In this limit the dispersion relation deformation can be parameterized quite generally, regardless of the explicit model under study. Due to the extremely large value of $M_{P} \simeq 10^{19} \mathrm{GeV} / \mathrm{c}^{2}$ a series expansion of $\delta E\left(p, m, M_{P}\right)$ in inverse powers of $M_{P}$ might prove useful along with the leading term in $1 / M_{P}$ as first approximation. Accordingly, $\delta E\left(p, m, M_{P}\right)$ would be written [15, 16] as

$$
\delta E\left(p, m, M_{P}\right) \simeq \frac{1}{2 M_{P}}\left(\eta_{1} p^{2}+\eta_{2} m c p+\eta_{3} m^{2} c^{2}\right)
$$

with the three real parameters: $\eta_{1}$ associated with the leading term, $\eta_{2}$ to the next leading term, and $\eta_{3}$ to the next-to-next leading term. As pointed out in many studies [14, 17, 20, astrophysical data could be sensitive to a leading-order deformation with $\left|\eta_{1}\right| \lesssim 1$. A preliminary analysis of the Fermi Space Telescope data 21 25] is currently underway to constrain $\eta_{1}$. Very recently [15, 16, constraining the functional form of (1) in the nonrelativistic (NR) regime $p \ll m c$ based on ultraprecise cold-atom-recoil-frequency experiments was proposed. In the NR limit, a more appropriate parametrization of $\delta E\left(p, m, M_{P}\right)$ is [15, 16]

$$
\delta E\left(p, m, M_{P}\right) \simeq \frac{1}{2 M_{P}}\left(\xi_{1} m c p+\xi_{2} p^{2}+\xi_{3} \frac{p^{3}}{m c}\right)
$$

with parameter bounds obtained as $-6.0<\xi_{1}<2.4$ for the leading-order deformation parameter and $-3.8 \times 10^{9}<$ $\xi_{2}<1.5 \times 10^{9}$ for the next-to-leading order, both within a $95 \%$ confidence level [15, 16.

The low- and high-energy bounds just mentioned are perfectly complementary. To illustrate this fact, consider the following deformation $\delta E\left(p, m, M_{P}\right)=$ $-\eta\left[m^{3} c^{4} / \sqrt{m^{2} c^{4}+p^{2} c^{2}}-m^{2} c^{2}\right] / M_{P}$ [15]. In the lowenergy NR limit this dispersion relation corresponds to (44) with $\xi_{2}=-\eta, \xi_{1}=\xi_{3}=0$, namely the next-to-leading order. In the high-energy UR limit, on the other hand, this corresponds to (3) with $\eta_{3}=2 \eta, \eta_{1}=\eta_{2}=0$ or to the next-to-next leading order. This example also exhibits how the the deformation $\delta E$ can be more important in the NR than in the UR limit.

It might be objected that (4) can be ruled out for macroscopic objects when $\xi_{1} \sim 1$. One has $p^{2} / 2 m \lesssim \delta E$ for $p \lesssim p_{0} \equiv \xi_{1} m^{2} c / M_{P}$ so that the deformation $\delta E$ dominates over the Minkowski kinetic term. Standard-model particles with $m \lesssim 10^{-16} M_{P}$ makes $\delta E$ dominate in the extreme NR limit $p \lesssim p_{0} \sim 10^{-16} \xi_{1} m c$. However, for macroscopic objects one can easily have $m \sim M_{P}$ and so that $p^{2} / 2 m \lesssim \delta E$ for $p \lesssim p_{0} \sim \xi_{1} m c$, i.e., the deformation $\delta E$ dominates in the entire NR regime. But this would contradict the familiar dynamics of classical NR bodies. Note, however, that (4) is merely the small- $p$ asymptotic expansion of the full deformation $\delta E\left(p, m, M_{P}\right)$, and is thus valid for all momenta up to some $p_{\lambda}$, where $p_{\lambda}$ depends on the explicit functional form of $\delta E$. For example a deformation

$$
\delta E\left(p, m, M_{P}, p_{\lambda}\right)=\xi_{1} \frac{m c p}{2 M_{P}} \exp \left(-p / p_{\lambda}\right)
$$

behaves as $\delta E=\xi_{1} m c p / 2 M_{P}$ for $p \lesssim p_{\lambda}$ and $\delta E \simeq 0$ for $p \gg p_{\lambda}$. Therefore, to apply (5) to macroscopic bodies one should measure the momenta of extended objects with $p \leq p_{\lambda}$ and this is impossible for sufficiently small $p_{\lambda}$ below the lowest measurable momentum for extended bodies. Since one supposes that this is always the case for $p_{\lambda}$, the relation (4) cannot be ruled out for classical macroscopic bodies. We also emphasize how (4) is commonly accepted in the literature [15, 16].

This Letter addresses the effect of a deformed dispersion relation on the critical temperature $T_{c}$ of a spatiallyuniform non-trapped Bose-Einstein condensate (BEC). This effect can be compared with the current precision in $T_{c}$ measurements that allows constraining the leading order parameter of the NR deformation (4) up to $\left|\xi_{1}\right| \lesssim 10^{2}$.

After languishing for seven decades as a mere academic exercise in textbooks, BEC was finally observed in the laboratory in laser-cooled, magnetically-trapped ultracold bosonic clouds of ${ }_{37}^{87} \mathrm{Rb}$ atoms [26], ${ }_{3}^{7} \mathrm{Li}$ [27, ${ }_{11}^{23} \mathrm{Na} 28,{ }_{1}^{1} \mathrm{H}$ 29], ${ }_{37}^{85} R b$ 30, ${ }_{2}^{4} \mathrm{He}$ [31], ${ }_{19}^{41} \mathrm{~K}$ [32], ${ }_{55}^{133} \mathrm{Cs}$ [33], ${ }_{70}^{174} \mathrm{Yb}$ 34] and ${ }_{24}^{52} \mathrm{Cr}$ 35. The relativistic BEC including antibosons as well as bosons has been reported [36], but for simplicity we neglect antibosons even in the UR limit in what follows. A generalization including antibosons is straightforward. Nowadays there exist very accurate measurements of BEC critical temperatures $T_{c}$ so that the aforementioned constraints can be addressed. Specifically, in Ref. 37. very accurate measurements of $T_{c}$ shifts due to strong interboson interactions are reported for ${ }_{19}^{39} K$. Such highprecision measurements might be useful in constraining Planck-scale dispersion relation deformations introduced in (11) in feasible experiments.

Neglecting interboson interactions in the BEC we find that the leading-order deformation in (4) produces a shift $\Delta T_{c} / T_{c}^{0} \propto \xi_{1} m^{2} c / \hbar M_{P} n^{1 / 3}$ where $\Delta T_{c} \equiv T_{c}-T_{c}^{0}$ with $T_{c}$ the critical temperature of the gas with the deformed dispersion relation, $T_{c}^{0}$ that same temperature in the undeformed Minkowski case, $n$ the boson number density and $m$ the boson mass. This shift can be unexpectedly high. For example, we obtain $\Delta T_{c} / T_{c}^{0} \sim 10^{-4} \xi_{1}$ for ${ }_{37}^{85} R b$ with a particle number density $n \simeq 10^{12} \mathrm{~cm}^{-3}$. Since in high-precision measurements of $\Delta T_{c} / T_{c}^{0}$ in ${ }_{19}^{39} \mathrm{~K}$ due to interboson interactions is $\Delta T_{c} / T_{c}^{0} \sim 5 \times 10^{-2}$ within a $1 \%$ error [37, the deformation parameter can be constrained up to $\left|\xi_{1}\right| \lesssim 10^{2}$. Moreover, since $\Delta T_{c} / T_{c}^{0} \propto n^{-1 / 3}$ one could enlarge the temperature shift merely by reducing $n$ (without however making it so small that it invalidates the thermodynamic limit), and therefore the bound on the deformation parameter $\xi_{1}$ can be improved using dilute (small $n$ ) BECs.

Based on such an unexpected relevant result, it behooves one to propose experiments with dilute BECs with the aim of measuring a Planck-scale induced shift of $T_{c}$. Of course, one should eventually generalize our result con- 
cerning $\Delta T_{c} / T_{c}^{0}$ to trapped BECs but this is not trivial. The main task there would be to calculate the energy levels of the trapped bosons that in the case of free bosons corresponds to the deformed dispersion relation (4), with the result expected to depend on the specific quantumgravity framework used. Additionally, in planning a feasible experiment one should include effects currently measurable experimentally 37] due to interboson interactions and compare them Planck-scale contributions to $\Delta T_{c} / T_{c}^{0}$. These caveats are currently under scrutiny 38 (see also refs. 39, 40).

We also analyze the effect of the next-to-leading-term deformation in (41) and show that one obtains $\Delta T_{c} / T_{c}^{0}=$ $\xi_{2} m / M_{P}$ which, being an extremely small and constant shift, cannot constrain the deformation parameter $\xi_{2}$ with $T_{c}$ measurements alone. Therefore, the next-to-leadingorder deformation in (4) cannot be excluded nor even bounded. Lastly, we derive the effect of a Planck-scale dispersion relation deformation in the UR limit (3) and find the remarkable result that this effect is always negligibly small. All this suggests that Planck-scale corrections to $T_{c}$ can be important for NR BECs rather than UR ones.

The general procedure to calculate $\Delta T_{c} / T_{c}^{0}$ is first sketched. We write the general form

$$
\delta E(p)=x f(p)
$$

for the dispersion relation deformation $\delta E(p)$ where $x \ll$ 1 is a dimensionless deformation parameter. For the dispersion-relation deformation defined in (6) the condensation temperature $T_{c}(x)$ is a function of the deformation parameter $x$ and is obtained by extracting $T_{c}(x)$ from its defining implicit relation

$2 \pi^{2} \hbar^{3} n=\int_{p_{0}}^{\infty}\left[\exp \left[\frac{E_{0}(p)+x f(p)-m c^{2}}{k_{B} T_{c}(x)}\right]-1\right]^{-1} p^{2} d p$.

where $p_{0} \equiv \pi \hbar / L$ for free particles in a box of volume $L^{3}$. Clearly, for $x=0$ one recovers the usual Minkowski value $T_{c}(0)=T_{c}^{0}$ that in the NR limit $E_{0}(p) \simeq m c^{2}+p^{2} / 2 m$ gives the familiar BEC formula

$$
T_{c}^{0}=T_{c}^{N R} \equiv \frac{2 \pi}{\zeta(3 / 2)^{2 / 3}} \frac{\hbar^{2} n^{2 / 3}}{k_{B} m} .
$$

Since the lhs of (77) is independent of $x$ one has $\partial_{x} n=0$. After some algebra one readily obtains

$$
\begin{aligned}
& \partial_{x} T_{c}(x) / T_{c}(x)=\int_{p_{0}}^{\infty} f(p) g(p, x) p^{2} d p / \int_{p_{0}}^{\infty} p^{2} d p \times \\
& {\left[E_{0}(p)+x f(p)-m c^{2}\right] g(p, x)}
\end{aligned}
$$

where we have defined

$$
\begin{aligned}
& g(p, x) \equiv\left(\exp \left[\frac{E_{0}(p)+x f(p)-m c^{2}}{k_{B} T_{c}(x)}\right]-1\right)^{-2} \times \\
& \exp \left[\frac{E_{0}(p)+x f(p)-m c^{2}}{k_{B} T_{c}(x)}\right] .
\end{aligned}
$$

This expression is quite useful to calculate the shift in $T_{c}$ due to the dispersion relation deformation. In fact, since $x \ll 1$, one can take

$$
\frac{\Delta T_{c}}{T_{c}^{0}}=\frac{T_{c}(x)-T_{c}(0)}{T_{c}(0)} \simeq x\left(\frac{\partial_{x} T_{c}(x)}{T_{c}(x)}\right)_{\mid x=0}
$$

and the last term can be evaluated by use of (9). Let us consider the leading term of the NR deformation given in (44), i.e., $\delta E=\xi_{1} m c p / 2 M_{P}$. This corresponds to $x=$ $\xi_{1} m / 2 M_{P}$ and $f(p)=c p$. In the NR limit one can write $E_{0}(p) \simeq m c^{2}+p^{2} / 2 m$ and $T_{c}(0)=T_{c}^{N R}$, so that (11) becomes

$$
\frac{\Delta T_{c}}{T_{c}^{0}} \simeq 0.1\left(\frac{m^{2} c}{\hbar M_{P} n^{1 / 3}}\right) \xi_{1} \ln (N) .
$$

where $N \equiv n L^{3}$ is the total number of particles. This shift can be evaluated for a ${ }_{37}^{85} R b$ BEC with number density $n \simeq 10^{12} \mathrm{~cm}^{-3}$ 41, boson mass $m \simeq 150 \times 10^{-27} \mathrm{~kg}$ and $N=10^{9}$. Hence $\Delta T_{c} / T_{c}^{0} \simeq 8.6 \times 10^{-5} \xi_{1}$. Since one expects $\xi_{1} \sim 1$ in quantum-gravity theories, this can be extremely large when compared to the strength of the deformation in (4) which is of order $\delta E / E \simeq \xi_{1} p / 2 c M_{P} \ll$ $\xi_{1} m / M_{p} \sim 10^{-17}$. Evidently, the temperature shift (12) being $\propto n^{-1 / 3}$ can be enlarged for BECs with sufficiently small $n$, but not so small to render the thermodynamic limit inapplicable. This fact is vital since one can then seek low-density BECs with a correspondingly large $\Delta T_{c} / T_{c}^{0}$ in order to constrain and perhaps even measure the deformation parameter $\xi_{1}$. Moreover, from (12) another way of enlarging $\Delta T_{c} / T_{c}^{0}$ is to consider more massive bosons.

As mentioned initially, if one is to deal with actual laboratory measurements of $\Delta T_{c} / T_{c}^{0}$ one should generalize (12) to the case of a trapped gas. However, such a generalization is a delicate matter well beyond the intent of this paper and that will be presented elsewhere [38. Here we merely stress that, for non-trapped BECs the shift $\Delta T_{c} / T_{c}^{0}$ due to Planck-scale effects can be as large as $\sim 10^{-4} \xi_{1}$ and even larger for dilute BECs. Since we expect a comparable effect for trapped BECs, it makes sense to compare such a shift with empirical values of $\Delta T_{c} / T_{c}^{0}$.

In high-precision measurements of $\Delta T_{c} / T_{c}^{0}$ in ${ }_{19}^{39} K[37$ due to interboson interactions, the order of magnitude is $\Delta T_{c} / T_{c}^{0} \simeq 5 \times 10^{-2}$ with at most a $1 \%$ error so that such measurements may be sensitive to Planck-scale effects. As seen above, the Planck-scale-induced shift in the condensation temperature is $\Delta T_{c} / T_{c}^{0} \simeq 10^{-4} \xi_{1}$ for a ${ }_{37}^{85} R b \mathrm{BEC}$, which allows to constrain the deformation parameter up to $\left|\xi_{1}\right| \lesssim 10^{2}$. Moreover, since the temperature shift can be enhanced for even more dilute and/or more massive BECs, even better bounds on $\xi_{1}$ are obtainable.

Note also that (12) may theoretically suggest the exclusion of the leading term in (4) as it would cause an unbounded shift for very small $n$. Even though the temperature shift becomes arbitrarily large for small $n$ the critical temperature vanishes as $n^{1 / 3}$ when $n \rightarrow 0$, since one has 


$$
T_{c} \simeq \frac{2 \pi}{\zeta(3 / 2)^{2 / 3}} \frac{\hbar^{2} n^{2 / 3}}{k_{B} m}\left[1+30\left(\frac{m^{2} c}{\hbar M_{P} n^{1 / 3}}\right) \xi_{1}\right] .
$$

Thus, a nonzero deformation parameter $\xi_{1}$ is not inconsistent. Remarkably therefore, a measured nonzero shift of $T_{c}$ in a low-density BEC, but unrelated to interboson interactions, would imply a leading-order dispersion relation deformation.

Let us briefly examine the next-to-leading-order term in (4), corresponding to $\xi_{2} \neq 0$ and $\xi_{1}=\xi_{3}=0$ with the bound $\left|\xi_{2}\right| \leq 10^{9}$ [15, 16]. This implies the energy dispersion relation

$$
E \simeq m c^{2}+p^{2} / 2 m+\xi_{2} p^{2} / 2 M_{P} \equiv m c^{2}+p^{2} / 2 m^{\xi_{2}}
$$

where $m^{\xi_{2}} \equiv m M_{P} /\left(\xi_{2} m+M_{P}\right)$. This gives $T_{c}=$ $T_{c}^{0}\left[1+\xi_{2}\left(m / M_{P}\right)\right]$ so that the temperature shift becomes

$$
\frac{\Delta T_{c}}{T_{c}^{0}}=\xi_{2} \frac{m}{M_{P}} .
$$

Since $m / M_{P} \ll 1$ such a shift is extremely small and therefore the next-to-leading term in (44) cannot be excluded nor bounded even for very large values of $\xi_{2}$.

So far our focus has been limited to the NR limit of the deformed dispersion relation (44). We now show that Planck-scale corrections to $T_{c}$ are negligibly small as a result of the leading term in the UR limit (3). In this case $\delta E(p)=\eta_{1} p^{2} / 2 M_{P}$ and the temperature shift can be calculated via (9-11) with $x=\eta_{1} m / 2 M_{P}$ and $f(p)=$ $p^{2} / m$. Moreover, one has $E_{0}(p) \simeq c p$ and $T_{c}(0)=T_{c}^{U R}$ where $T_{c}^{U R}$ is the UR condensation temperature

$$
T_{c}^{U R} \equiv \hbar c \pi^{2 / 3} n^{1 / 3} / k_{B} \zeta(3)^{1 / 3} .
$$

The resulting temperature shift is thus

$$
\frac{\Delta T_{c}}{T_{c}^{0}} \simeq 1.8 \eta_{1}\left(\frac{k_{B} T_{c}^{U R}}{c^{2} M_{P}}\right)
$$

and such a shift can be appreciable only for extremely high densities $n$ such that $k_{B} T_{c}^{U R} \sim c^{2} M_{P} \sim 10^{19} \mathrm{GeV}$. This result is significant as being counterintuitive since one expects that Planck-scale effects be appreciable in UR rather than NR phenomena. In fact, the situation is quite the opposite: Planck-scale corrections to BEC critical $T_{c} \mathrm{~S}$ are appreciable in NR BECs (12) but are extremely small for UR BECs (17).

Lastly, we briefly discuss the cosmological consequences of our analysis. It was recently proposed [42 64 that dark matter in the universe might consist of a BEC phase due to some boson. Since the condensation temperature determines the epoch of formation of such a condensate, a shift in $T_{c}$ may delay or anticipate the condensation of darkmatter particles, thus affecting the phenomenology of the model. This motivates exploring whether Planck-scale deformation can in fact affect BECs. At any rate, one is far from a direct observation of such a cosmological condensate so that at present its existence is speculative, even though one can search for its indirect cosmological traces. Indeed, this suggests an interesting avenue of research.

To conclude, we have determined the effects of Planckscale deformation of the dispersion relation on the condensation temperature of a non-trapped BEC. In particular, assuming a nonrelativistic leading-order dispersion relation defined in (4) one finds that such an effect may be comparable with the precision measurement of $T_{c}$ 37. and that one can bound the leading-order deformation parameter up to $\left|\xi_{1}\right| \lesssim 10^{2}$. We thus argue that one should generalize this result to the case of trapped BECs [38] in order to propose feasible experiments sensitive to Planckscale physics. We have also shown that the next-to-leading correction in (4) causes an extremely small shift that is unobservable with any experiment so such a deformation can neither be excluded nor fixed outside presently existing bounds. Finally, it is noteworthy that Planck-scale corrections to $T_{c}$ are extremely small for UR condensates. This leads one to the conclusion that Planck-scale physics may be relevant for cold-atom NR BECs rather than UR BECs.

Acknowledgements: FB thanks G. Amelino-Camelia for useful discussions during the early stages of this Letter and for his continued encouragement since then. It was completed during a visit of FB at IIM-UNAM in Mexico City. FB and MdeLl thank UNAM-DGAPA-PAPIIT (México) for its support from grant IN106908.

\section{REFERENCES}

[1] G. Amelino-Camelia, Lect. Notes Phys. 541, 1 (2000).

[2] J. Alfaro, H.A. Morales-Tecotl, and L.F. Urrutia, Phys. Rev. Lett. 84, 2318 (2000).

[3] T. Jacobson, S. Liberati, and D. Mattingly, Lect. Notes Phys. 669, 101 (2005).

[4] G. Amelino-Camelia and C. Lämmerzahl, Class. Quant. Grav. 21, 899 (2004).

[5] U. Jacob and T. Piran, Nature Phys. 3, 87 (2007).

[6] F. Aharonian et al. [HESS Collaboration], Phys. Rev. Lett. 101, 170402 (2008).

[7] G. Amelino-Camelia, C. Lämmerzahl, F. Mercati, and G.M. Tino, Phys. Rev. Lett. 103, 171302 (2009).

[8] R. Gambini and J. Pullin, Phys. Rev. D 59, 124021 (1999).

[9] G. Amelino-Camelia and S. Majid, Int. J. Mod. Phys. A 15, 4301 (2000).

[10] G. Amelino-Camelia, F. Briscese, G. Gubitosi, A. Marciano, P. Martinetti, and F. Mercati. Phys. Rev. D 78, 025005 (2008).

[11] J. Kowalski-Glikman, Phys. Lett. B 499, 1 (2001).

[12] O. Bertolami and L. Guisado, JHEP 0312, 013 (2003).

[13] S.R. Coleman and S.L. Glashow, Phys. Rev. D 59, 116008 (1999).

[14] G. Amelino-Camelia, J. Ellis, N.E. Mavromatos, D.V. Nanopoulos, and S. Sarkar, Nature 393, 763 (1998).

[15] G. Amelino-Camelia, C. Lämmerzahl, F. Mercati, and G.M. Tino, Phys. Rev. Lett. 103,171302 (2009). 
[16] F. Mercati, D. Mazon, G. Amelino-Camelia, J.M. Carmona, J.L. Cortés, J. Induráin, C. Lämmerzahl, and G.M. Tino, Class. Quant. Grav. 27, 215003 (2010).

[17] B.E. Schaefer, Phys. Rev Lett. 82, 4964 (1999).

[18] S.D. Biller, A.C. Breslin, J. Buckley, M. Catanese, M. Carson, D.A. Carter-Lewis, M.F. Cawley, D.J. Fegan, J.P. Finley, J.A. Gaidos, A.M. Hillas, F. Krennrich, R.C. Lamb, R. Lessard, C. Masterson, J.E. McEnery, B. McKernan, P. Moriarty, J. Quinn, H.J. Rose, F. Samuelson, G. Sembroski, P. Skelton, and T.C. Weekes, Phys. Rev. Lett. 83, 2108 (1999).

[19] T. Kifune, Astrophys. J. Lett. 518, L21 (1999).

[20] G. Amelino-Camelia, Nature 408, 661 (2000).

[21] A.A. Abdo, M. Ackermann, et al., Science 323, 1688 (2009)

[22] J. Ellis, N.E. Mavromatos, and D.V. Nanopoulos, Phys. Lett. B 674, 83 (2009).

[23] G. Amelino-Camelia and L. Smolin, Phys. Rev. D 80, 084017 (2009).

[24] A.A. Abdo et al., Nature 462, 331 (2009).

[25] G. Amelino-Camelia, Nature 462, 291 (2009).

[26] M.H. Anderson, J.R. Ensher, M.R. Wieman, and E.A. Cornell, Science 269, 198 (1995).

[27] C.C. Bradley, C.A. Sackett, J.J. Tollett, and R.G. Hulet, Phys. Rev. Lett. 75, 1687 (1995).

[28] K.B. Davis, M.O. Mewes, M.R. Andrews, N.J. van Drutten, D.S. Durfee, D.M. Kurn, and W. Ketterle, Phys. Rev. Lett. 75, 3969 (1995).

[29] D.G. Fried, T.C. Killian, L. Willmann, D. Landhuis, S.C. Moss, D. Kleppner, and T.J. Greytak, Phys. Rev. Lett. 81, 3811 (1998).

[30] S.L. Cornish, N.R. Claussen, J.L. Roberts, E.A. Cornell, and C.E. Wieman, Phys. Rev. Lett. 85, 1795 (2000).

[31] F. Pereira Dos Santos, J. Léonard, Junmin Wang, C.J. Barrelet, F. Perales, E. Rasel, C.S. Unnikrishnan, M. Leduc, and C. Cohen-Tannoudji, Phys. Rev. Lett. 86, 3459 (2001).

[32] G. Modugno, G. Ferrari, G. Roati, R.J. Brecha, A. Simoni, and M. Inguscio, Science 294, 1320 (2001).

[33] T. Weber, J. Herbig, M. Mark, H.C. Nägerl, and R. Grimm, Science 299, 232 (2003).

[34] Y. Takasu, K. Maki, K. Komori, T. Takano, K. Honda, M. Kumakura, T. Yabuzaki, and Y. Takahashi, Phys. Rev. Lett. 91, 040404 (2003).

[35] A. Griesmaier, J. Werner, S. Hensler, J. Stuhler, and T. Pfau, Phys. Rev. Lett. 94, 160401 (2005).

[36] M. Grether, M. de Llano, and G.A. Baker, Jr., Phys. Rev. Lett. 99, 200406 (2007).

[37] R.P. Smith, R.L.D. Campbell, N. Tammuz, and Z. Hadzibabic, Phys. Rev. Lett. 106, 250403 (2011).

[38] F. Briscese, arXiv:1206.1236v1 [gr-qc].

[39] E. Castellanos and C. Laemmerzahl, arXiv:1202.3801 1 [math-ph].

[40] E. Castellanos and C. Laemmerzahl, arXiv:1202.3806 1 [cond-mat.stat-mech].

[41] M. Grether, M. de Llano, S. Ramírez, and O. Rojo, Int. J. Mod. Phys. B 22, 4367 (2008), see esp. Table 3.

[42] A.P. Lundgren, M. Bondarescu, R. Bondarescu, and J. Balakrishna, Astrophys. J. 715, L35 (2010).

[43] I. Rodriguez-Montoya, J. Magaña, T. Matos, and A.Pérez-Lorenzana, Astrophys. J. 721, 1509 (2010).

[44] T.P. Woo and T. Chiueh, Astrophys. J. 697, 850 (2009).
[45] L.A. Ureña-López, JCAP 0901, 014 (2009).

[46] S. Fagnocchi, S. Finazzi, S. Liberati, M. Kormos, and A. Trombettoni. New J. Phys. 12, 095012 (2010).

[47] T. Harko, Monthly Not. Roy. Astron. Soc. 413, 3095 (2011).

[48] A. Suárez and T. Matos, arXiv:1101.4039v2 [gr-qc].

[49] T. Harko and F.S.N. Lobo, Astroparticle Physics 35 (2012) pp. 547-551.

[50] L.A. Gergely, T. Harko, M. Dwornik, G. Kupi, and Z. Keresztes, Mon. Not. Royal Astron. Soc. 415:3275-3290 (2011).

[51] T. Harko, Phys. Rev. D 83, 123515 (2011).

[52] T. Harko, Mon. Not. Roy. Astron. Soc. 413 (2011) 3095.

[53] P.H. Chavanis, Phys.Rev. D 84, 043531 (2011).

[54] J. Barranco, A. Bernal, J.C. Degollado, A. Diez-Tejedor, M. Megevand, M. Alcubierre, D. Núñez, and O. Sarbach, arXiv:1108.0931v1 [gr-qc].

[55] J. Barranco and A. Bernal, arXiv:1108.1208v1 [astroph.CO].

[56] T. Harko and E.J.M. Madarassy, JCAP 01 (2012) 020.

[57] T. Harko and F.S.N. Lobo, Phys.Rev.D83:124051,2011.

[58] T. Matos and A. Suárez, arXiv:1110.3114v1 [gr-qc].

[59] I. Rodríguez-Montoya, A. Perez-Lorenzana, E. De La Cruz-Burelo, Y. Giraud-Heraud, and T. Matos, arXiv:1110.2751v1 [astro-ph.CO].

[60] T. Matos, J. Phys. Conf. Ser. 315:012025,2011.

[61] T. Matos, A. Suárez, arXiv:1103.5731 4 [gr-qc].

[62] J. Magaña, T. Matos, V. Robles, and A. Suárez, arXiv:1201.6107 v1 [astro-ph.CO].

[63] M. Arik, M. Calik, N. Katirci, Central Eur.J.Phys.9:1465$1471,2011$.

[64] F. Briscese, Phys. Lett. B 696, 315 (2011). 